\title{
Single-direction thoracoscopic basal segmentectomy
}

Chengwu Liu, MD, Hu Liao, MD, Chenglin Guo, MD, Qiang Pu, MD, Jiandong Mei, MD, and Lunxu Liu, MD, PhD

\section{ABSTRACT}

Objective: Thoracoscopic anatomic single or combined basal segmentectomy is technically challenging because of the variation and deep location of vessels and bronchi in the parenchyma. This study aimed to describe thoracoscopic segmentectomy of basal segments using a single-direction method.

Methods: This retrospective study included 137 patients who underwent single or combined thoracoscopic basal segmentectomy between April 2015 and August 2019. All procedures were performed via the preferred inferior pulmonary ligament approach or an interlobar fissure approach following a single-direction strategy.

Results: Ninety patients underwent single basal segmentectomy, and 47 patients underwent combined basal segmentectomy. Median operative time was 125 minutes (range, 52-237 minutes), and median blood loss was $30 \mathrm{~mL}$ (range, 5-250 mL). Median chest tube duration was 2 days (range, 1-22 days), and median postoperative hospital stay was 4 days (range, 2-24 days). The postoperative morbidity rate was $5.1 \%$ (7/137). No perioperative deaths were identified. Pathological examination revealed 133 cases of lung cancer, 2 cases of metastasis, and 2 cases of benign tumors. No recurrence or mortality was observed during the median follow-up period of 15 months (range, 1-53 months).

Conclusions: The single-direction method for thoracoscopic single or combined basal segmentectomy was feasible and safe in our experience. This method exposes the targeted vessels and bronchi from superficial to deep in order of their appearance and enables anatomic resection of a single segment or combined basal segments to be performed in a simple manner while avoiding dissection of a hypoplastic fissure or inessential splitting of the lung parenchyma. ( $\mathrm{J}$ Thorac Cardiovasc Surg 2020;160:1586-94)

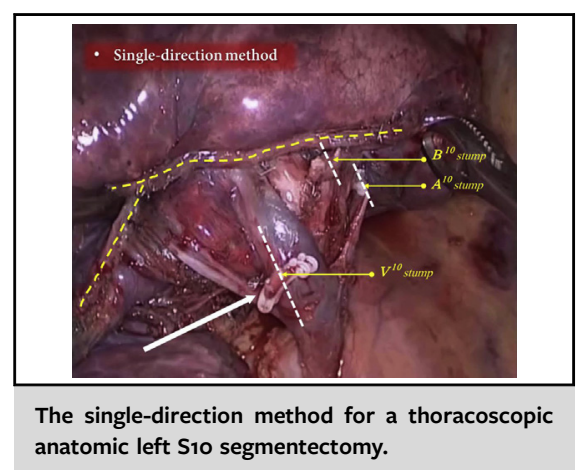

CENTRAL MESSAGE

The single-direction method enables thoracoscopic single or combined basal segmentectomy to be performed in a simple manner.

\section{PERSPECTIVE \\ Thoracoscopic single or combined basal segmentectomy is technically challenging. The single-direction method exposes the targeted components from superficial to deep in order of their appearance and allows the performance of a single or combined basal segmentectomy in a simple manner while avoiding dissection of a hypoplastic fissure or inessential splitting of the lung parenchyma.}

See Commentaries on pages 1595 and 1596.
Low-dose computed tomography (CT) screening has been reported to reduce mortality from lung cancer. ${ }^{1}$ Since then, CT has been widely used for lung cancer screening, enabling the detection of an increasing number of small-sized lung cancers. Although lobectomy is still the standard treatment for early-stage lung cancer, anatomic segmentectomy is indicated more frequently with comparable long-term

From the Department of Thoracic Surgery, West China Hospital, and Western China Collaborative Innovation Center for Early Diagnosis and Multidisciplinary Therapy of Lung Cancer, Sichuan University, Chengdu, China.

Drs C. Liu and H. Liao contributed equally to this article.

This study is supported by the Basic Science Program (2019YJ0077 to C.L.), Science and Technology Department of Sichuan Province, and 1.3.5 Project for Disciplines of Excellence (ZYJC18009 to J.M. and ZYGD18021 to L.L.), West China Hospital, Sichuan University. outcomes, especially for nonsolid tumors with ground-glass opacity (GGO). ${ }^{2-6}$ It is also realistic to expect that ongoing prospective, randomized studies (CALGB-140503 and JCOG-0802) will provide a specific conclusion for the optimal treatment. $^{7,8}$

Anatomic segmentectomy is a more complex procedure than lobectomy, and it is challenging and technique

Received for publication Oct 29, 2019; revisions received Jan 4, 2020; accepted for publication Jan 7, 2020; available ahead of print Feb 1, 2020.

Address for reprints: Lunxu Liu, MD, PhD, No. 37, Guoxue Alley, Chengdu, Sichuan, 610041, China (E-mail: lunxu_liu@aliyun.com).

0022-5223

Copyright (C) 2020 The Author(s). Published by Elsevier Inc. on behalf of The American Association for Thoracic Surgery. This is an open access article under the CC BY-NC-ND license (http://creativecommons.org/licenses/by-nc-nd/4.0/). https://doi.org/10.1016/j.jtcvs.2020.01.028 


\section{Abbreviations and Acronyms}

$\mathrm{CT}=$ computed tomography

GGO = ground-glass opacity

HRCT $=$ high-resolution computed tomography

Scanning this $\mathrm{QR}$ code will take

you to the article title page to

access supplementary information.

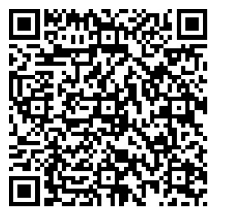

demanding, especially when performed under total thoracoscopic visualization. The main reason for its challenges lies in the difficulty of dissecting the frequently variable segmental structures (veins, arteries, and bronchi) and identifying the accurate intersegmental planes. ${ }^{9}$ Single or combined basal segmentectomies (7-10) are more technically challenging than other types of segmentectomies because of the deeper intraparenchymal localization of the hilar structures, more frequent variations, and more complex neighboring relationships of the intersegmental planes. Consequently, the published reports on complete thoracoscopic single or combined basal segmentectomies are limited by their small sample sizes. ${ }^{10-14}$ Conventionally, the surgical approaches for basal segments have been designed from the interlobar fissure, posterior approach, or bidirectional approaches. ${ }^{11,12,14}$ Surgeons need to split the pulmonary parenchyma or dissect a hypoplastic interlobar fissure. In addition, these methods are not carried out with single S9 segmentectomy or S8 segmentectomy with hypoplastic fissures. We developed the concept of single direction for thoracoscopic lobectomy ${ }^{15}$ and applied it to thoracoscopic basal segmentectomy through an inferior pulmonary ligament approach. ${ }^{16}$ In addition, we developed the method of "stem-branch" for tracking the target segmental structures. ${ }^{17}$

The objective of this study was to report our experience in thoracoscopic anatomic single or combined segmentectomy using the single-direction method and to determine the feasibility of this method on the basis of the largest case series performed until now.

\section{PATIENTS AND METHODS \\ Patients}

Between April 2015 and August 2019, a total of 137 patients underwent thoracoscopic single or combined basal segmentectomy following the singledirection strategy in our department. Patients' data were prospectively collected and retrospectively reviewed. Written informed consent was obtained from each patient before surgery. The protocol of this study was approved by the institutional review board of West China Hospital (NO. 2019-909).

\section{Selection Criteria}

The specific candidates are as follows: patients with a $2 \mathrm{~cm}$ or less GGO dominant (GGO $\geq 50 \%$ ) peripheral pulmonary nodule that might be lung cancer, patients with a pulmonary metastasis, or patients with an indeterminate lung lesion that might be benign. Patients who are not suitable for lobectomy due to compromised cardiopulmonary function are also appropriate candidates for this parenchyma-sparing procedure. It should be noted that only patients with lesions $1 \mathrm{~cm}$ or less, GGO $75 \%$ or greater, and $2 \mathrm{~cm}$ or less away from the visceral pleura were chosen as potential candidates for intentional wedge resection in our center. However, segmentectomy was also a preferred procedure for such cases. The surgical margin was designed to be no less than $2 \mathrm{~cm}$ or larger than the maximum diameter of the tumor. In cases with the lesion located too close to the intersegmental border, a combined segmentectomy was performed. The specimen was examined immediately after resection. If the surgical margin was insufficient, an additional wedge resection was performed. In cases of pulmonary adenocarcinoma, the new proposed histologic classification system ${ }^{18}$ and the eighth edition of the TNM staging system $^{19}$ were adopted for histologic typing and surgical-pathologic staging, respectively.

\section{OPERATIVE PROCEDURE Preoperative Planning}

Preoperative evaluation included regular blood tests, cardiopulmonary function tests, and imaging studies of the brain, upper abdomen, and the bone. Contrastenhanced high-resolution CT (HRCT) scans were routinely performed and carefully reviewed. The anatomic variations and positional relationships of the basal segmental vessels and bronchi were analyzed (Figure 1, $A$ and $B$, and Video 1). The location and radiologic features of the target nodules were identified. Furthermore, data on the anatomic relationship between the nodules and the neighboring structures were collected to design an appropriate surgical excision. Sometimes, in cases where the nodules were $7 \mathrm{~mm}$ or less, preoperative CT-guided hook-wires were used for localization.

\section{Surgical Techniques}

The patient was administered with general anesthesia and differential ventilation. The position and port strategy are shown in Figure 2. Digital palpation was often used to localize the target tumor, and marking directly on the surface of the visceral pleura was performed using sutures. ${ }^{20,21}$ All procedures were performed proceeding in a single-direction strategy through an interlobar fissure approach or an inferior pulmonary ligament approach. The inferior pulmonary ligament approach was preferred with no need to consider whether the interlobar fissures were complete or not. However, in cases of complete interlobar fissures, segmentectomies for S7, S8, or both could also be performed in a single-direction manner via an interlobar fissure approach.

As we have already described the detailed procedures of $\mathrm{S} 9^{17}$ and $\mathrm{S} 9+10^{16}$ in previous reports, we used a left $\mathrm{S} 10$ segmentectomy as an example to depict the detailed techniques in this study (Video 2). The surgery was initiated 


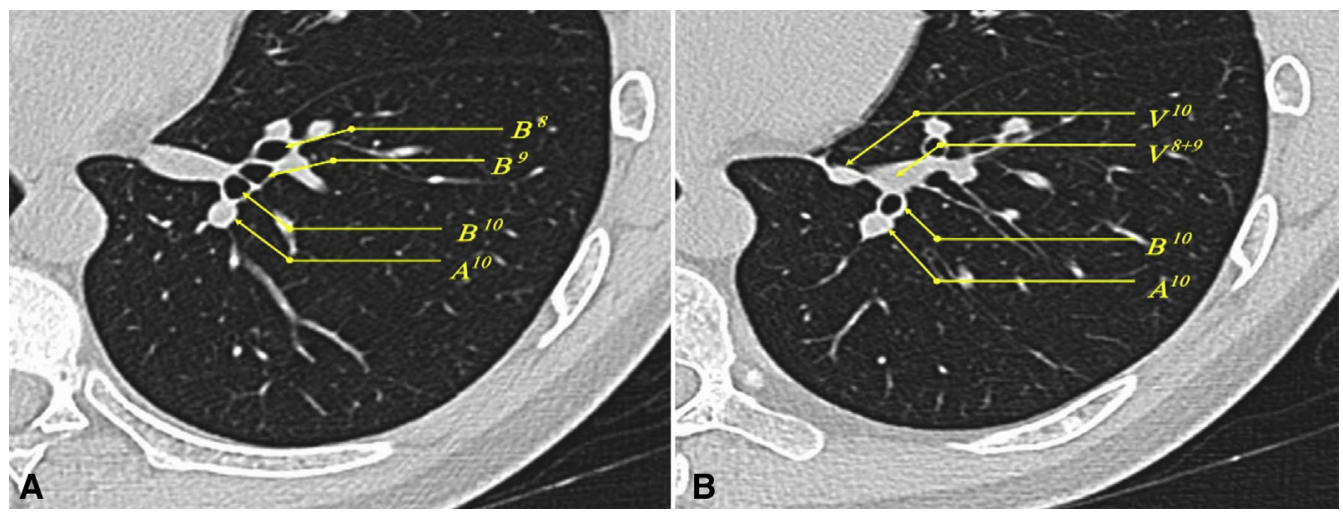

FIGURE 1. Identifying the target segmental vessels and bronchi and their positional relationships with other basal segmental structures (a left S10 segmentectomy as an example). A, The 3 branches of the common basal bronchus (B8, B9, and B10) arise at almost the same horizontal level. The most posterior branch is $\mathrm{B} 10$. A10 running along B10. B, V10 running alone while V8 and V9 converge in this section. B8. Bronchus of the anterior basal segment. B9. Bronchus of the lateral basal segment. B10. Bronchus of the dorsal basal segment. A10. Artery of the dorsal basal segment. V8+9. Veins of the anterior and lateral basal segments. V10. Vein of the dorsal basal segment.

by incising the inferior pulmonary ligament with retraction of the lower lobe toward the cranial side. The inferior pulmonary vein and its branches were first dissected. The drainage vein of S6 was identified first; then, dissection and identification of the common basal vein and its branches were carried out by referring to the preoperative imaging study (Figure 3,A). The drainage vein, which entered the target segment directly and with the highest tension when retracting the target segment, was divided while preserving the intersegmental veins. From an inferior view, the lower lobe bronchus was located just behind the inferior pulmonary vein. The stem and branches of the common basal segmental bronchus were usually dissected

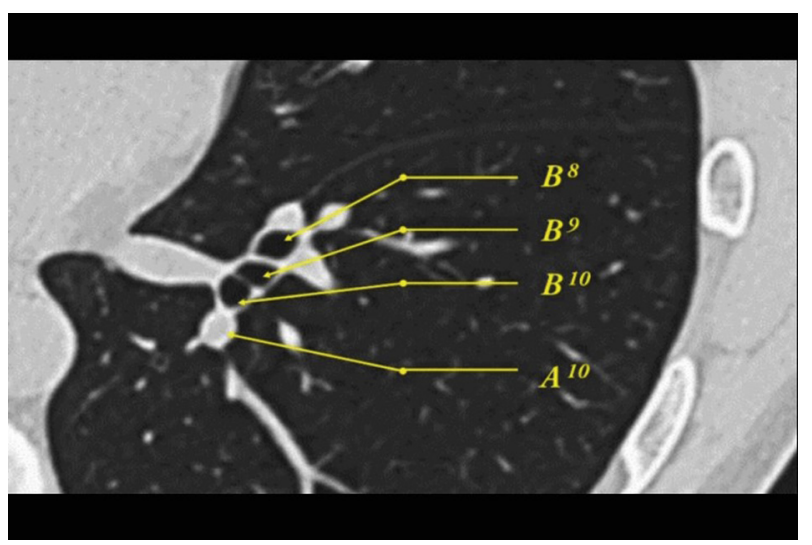

VIDEO 1. Analyzing the anatomic variations and positional relationships of the basal segmental vessels and bronchi by carefully reviewing the HRCT images (a left S10 segmentectomy as an example). B8. Bronchus of the anterior basal segment. B9. Bronchus of the lateral basal segment. B10. Bronchus of the dorsal basal segment. A10. Artery of the dorsal basal segment. V8+9. Veins of the anterior and lateral basal segments. V10. Vein of the dorsal basal segment. Video available at: https://www.jtcvs.org/ article/S0022-5223(20)30249-X/fulltext. from proximal to distal along the stem. At the same time, peribronchial lymph nodes were retrieved. The target segmental bronchus was tracked and identified using the "stem-branch" method (Figure 3, B). During this process, the stem was first identified. Then, the bifurcations of its branches were dissected and identified by tracking along the stem from proximal to distal. Finally, the target bronchus was identified by referring to the anatomic features already known from the preoperative HRCT. The target bronchus was clamped and further confirmed via inflation of the lung on the operating side. After dividing the target segmental bronchus, the target segmental feeding artery always came directly into sight because it was always running along the bronchus (Figure 3,C). After dividing the artery, the last step was the management of the intersegmental planes. The intersegmental pulmonary parenchyma was further dissected along the intersegmental veins using energy devices. The method of inflationdeflation was used to show the intersegmental demarcation line. Then, the intersegmental planes were tailored using a stapler in a step-by-step fashion (Figure 3,D). Finally, a single-direction S10 segmentectomy was completed (Figure 4). During the interlobar fissure approach, the target artery, bronchus, vein, and intersegmental planes were managed sequentially upon their appearance in a single-direction manner.

The surgical margin was checked immediately. Both the tumor and station 13 lymph nodes were sent for intraoperative frozen-section pathological examinations. Hilar and mediastinal lymph node dissection was performed if primary pulmonary malignancy was confirmed. Lobe-specific mediastinal lymph node dissection (stations 7, 8, and 9) was sometimes performed for cases of GGO-dominant nodules. One chest tube was placed through the thoracoscope port. The incisions were closed 


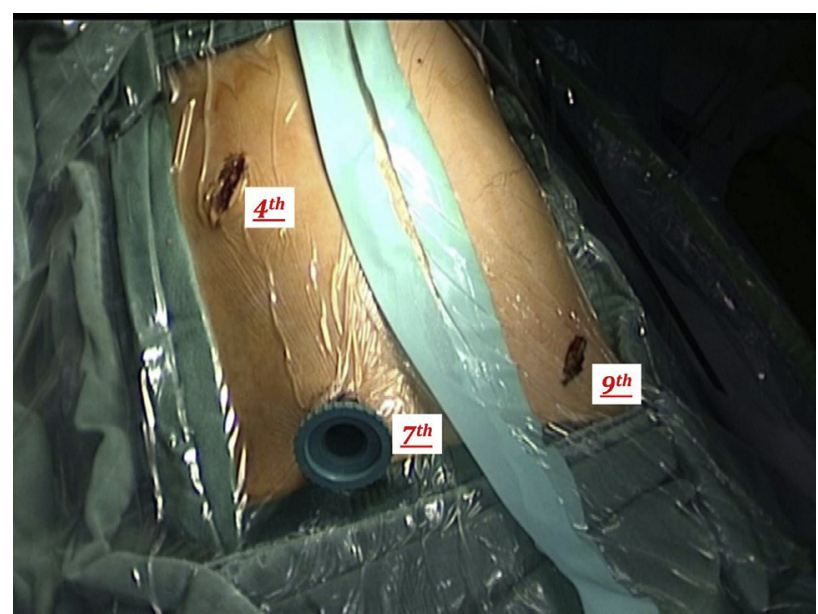

FIGURE 2. The position of the patient and port strategy used for thoracoscopic single or combined basal segmentectomy (left side). The patient was placed in the lateral decubitus position. The observation port $(1 \mathrm{~cm})$ in the seventh intercostal space (ICS) at the midaxillary line; the main utility incision $(3 \mathrm{~cm})$ in the fourth ICS at the anterior axillary line; the assistant incision $(2 \mathrm{~cm})$ in the ninth ICS between the posterior axillary line; and the subscapular line.

after careful examination for active bleeding and massive air leakage.

The chest tube was removed if there was no air leakage, it was well inflated lung on chest $x$-ray, and there was less than $300 \mathrm{~mL}$ of drainage during the last 24 hours. Patients were discharged home with no main complications. The last follow-up was in September 2019.

\section{Statistical Analysis}

The data were analyzed using SPSS, version 22.0 (SPSS Inc, Chicago, Ill).

\section{RESULTS}

The patient characteristics are shown in Table 1 . The different types of basal segmentectomies are listed in Table 2 . Ninety patients underwent single basal segmentectomy, and 47 patients underwent combined basal segmentectomy. Thirty-three cases were performed via an interlobar fissure approach, and the other 104 cases were performed via an inferior ligament approach. The most technically challenging type (single S9 segmentectomy) was successfully performed in 12 patients. Thirty-six patients underwent synchronous combined surgeries for multiple lesions in other lobes; 18 patients underwent wedge resection, 10 patients underwent segmentectomy, 4 patients underwent lobectomy, 2 patients underwent subsegmentectomy, 1 patient underwent lobectomy and wedge resection, and 1 patient underwent segmentectomy and wedge resection.

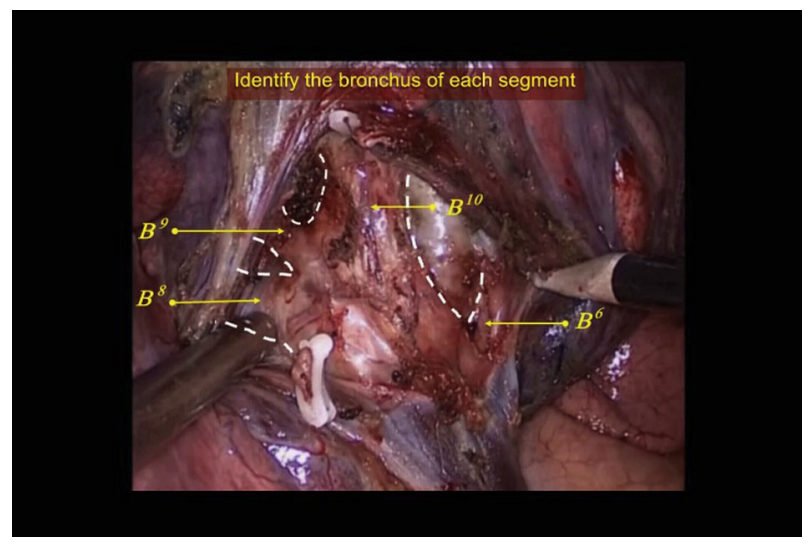

VIDEO 2. A single-direction video-assisted thoracic surgery left S10 segmentectomy through an inferior pulmonary approach. A 33-year-old man with no comorbidities was diagnosed with 3 GGO nodules located in the right upper lobe $(8 \mathrm{~mm})$, left upper lobe $(6 \mathrm{~mm})$, and left lower lobe $(6 \mathrm{~mm})$. He underwent right upper lobe wedge resection and lymph node sampling 6 months before this operation with a final pathological diagnosis of minimally invasive adenocarcinoma with no lymph node metastasis. Because the nodule in the left lower lobe was deeply located (Video 1), wedge resection seemed not to be appropriate. Wedge resection was performed first for the left upper nodule. Then, S10 segmentectomy was initiated with dissection of the inferior pulmonary ligament and the inferior pulmonary vein and its branches, with the lower lobe retracted toward the cranial side. V6 was first isolated, and then the common basal segmental vein could be confirmed. Along with the stem of the common basal vein, the branches were tracked and V10 was identified easily according to the preoperative CT scan. Thereafter, the common basal bronchus and its branches were exposed and dissected. The most important step of this procedure was to track along the stem and find the bifurcations of the 3 branches. B 10 could be easily identified because we already knew the anatomic features of these branches. As shown in Figure 1, A, they arouse at almost the same level, and the most posterior branch was B10. After dividing the bronchus, A10 was easy to find and was divided without concern because we knew that A10 was located just behind B10 and that there was no variation. The nodule was slightly close to the intersegmental border between S6 and S10, so we performed an intentional extended segmentectomy to ensure a sufficient margin. A stapler-based technique was used for demarcation of the intersegmental planes to reduce the risk of postoperative air leakage. The key point during this process was to start from the most peripheral and thin parts of the lung and to continue to approach the segmental hilum and thick parts of the lung in a step-by-step procedure. The frozen-section pathologic examination confirmed minimally invasive adenocarcinoma and no metastasis to the peribronchial lymph nodes. Systemic hilar and mediastinal lymph node sampling was performed (because the procedure for lymph node sampling is easy, it is omitted in this video). The operative time was 130 minutes. The patient was discharged on postoperative day 4 . The CT scans 3 months after the operation revealed well-inflated lungs with no obvious atelectasis at the stapling sites. B8. Bronchus of the anterior basal segment. B9. Bronchus of the lateral basal segment. B10. Bronchus of the dorsal basal segment. A10. Artery of the dorsal basal segment. V8+9. Veins of the anterior and lateral basal segments. V10. Vein of the dorsal basal segment. VATS, Video-assisted thoracic surgery. Video available at: https://www. jtcvs.org/article/S0022-5223(20)30249-X/fulltext. 

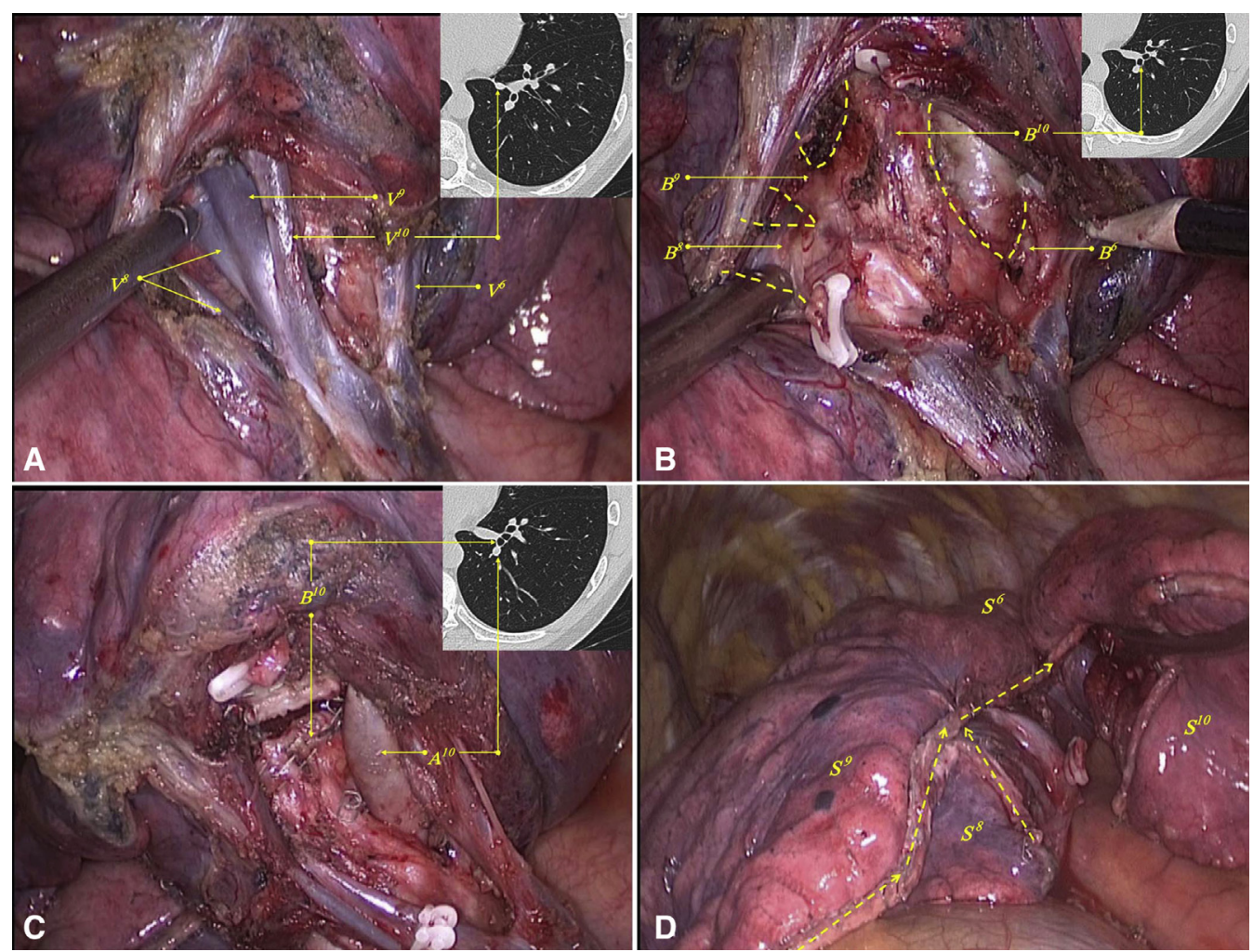

FIGURE 3. A single-direction video-assisted thoracoscopic surgery left S10 segmentectomy through an inferior pulmonary ligament approach. A, Dissecting the inferior pulmonary vein and all of the branches through the inferior pulmonary ligament approach. Identifying the venous branch of S10, which is an isolated branch, according to the preoperative CT scan. Then, the branch was clamped using hem-o-locks and divided using scissors. $\mathrm{B}$, The common basal bronchus and its branches were dissected just behind the pulmonary vein. The 2 bifurcations of the common basal segmental bronchus were identified. The 3 bronchial branches were confirmed to arise at almost the same location. B10, which is the most posterior of the 3 branches, was identified. Then, B10 was divided using a stapler. C, A10 was dissected and identified just posterior to B10. A10 runs along B10. A10 was clamped using hem-o-locks and divided using scissors. D, The intersegmental planes were identified using the method of inflation/deflation. The intersegmental planes were tailored using a stapler-based technique: First, S10 was split from S8; second, S10 was split from S9; third, S10 was split from the intersection among S6, S8, and S9; fourth, S10 was split from S6, completing the S10 segmentectomy. V8. Vein of the anterior basal segment. V9. Vein of the lateral basal segment. V10. Vein of the dorsal basal segment. B6. Bronchus of the superior segment. B8. Bronchus of the anterior basal segment. B9. Bronchus of the lateral basal segment. B10. Bronchus of the dorsal basal segment. A10. Artery of the dorsal basal segment. S6. Superior segment. S8. Anterior basal segment. S9. Lateral basal segment. S10. Dorsal basal segment.

All procedures were successfully performed under thoracoscopic visualization, and no conversion to lobectomy occurred. The perioperative outcomes are listed in Table 3. Median operative time was 125 minutes (range, 52-237 minutes), and median blood loss was $30 \mathrm{~mL}$ (range, 5-250 mL). Median chest tube duration was 2 days (range, 1-22 days), and median postoperative hospital stay was 4 days (range, 2-24 days). The postoperative morbidity was $5.1 \%(7 / 137$, including 5 patients with prolonged air leakage $>5$ days and 2 patients with both prolonged air leakage $>5$ days and pneumonia). All patients were discharged with no obvious symptoms. There were no instances of perioperative death. When compared with patients who only underwent basal segmentectomy, the 36 patients who underwent combined surgeries had significantly longer operative time $(151.83 \pm 42.29$ minutes vs
$121.06 \pm 35.90$ minutes, $P<.001)$, longer chest tube duration (4.94 \pm 4.54 days vs $2.50 \pm 1.16$ days, $P<.001$ ), longer postoperative hospital stay $(6.50 \pm 4.54$ days vs $4.43 \pm 1.98$ days, $P<.001$ ), and higher postoperative morbidity $(6 / 30$ vs $1 / 100, P=.001)$, whereas the blood loss was comparable $(53.61 \pm 41.07 \mathrm{~mL}$ vs $41.09 \pm 41.10 \mathrm{~mL}, P=.119)$.

Final pathologic examination revealed that radical resections were achieved with free surgical margins in all patients. Twenty-five cases were confirmed with synchronous multiple primary lung cancers. Median size of the tumor in the resected basal segment was $1.0 \mathrm{~cm}$ (range, 0.5-2.7 cm). The histologic subtypes of the basal segmental lesions were as follows: 133 cases of primary lung cancer (132 cases of adenocarcinoma and 1 case of squamous cell carcinoma), 2 cases of metastatic lesions, 


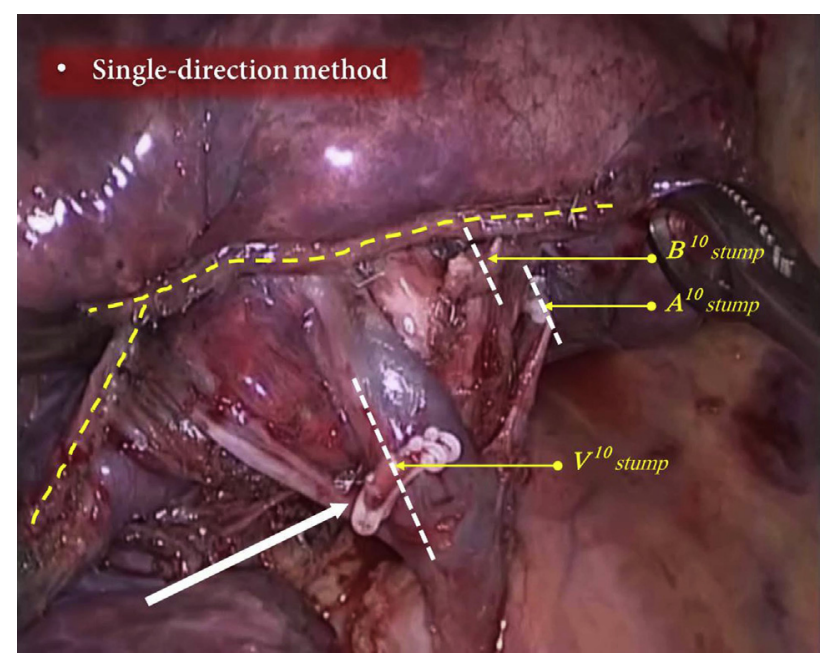

FIGURE 4. Demonstration of the single-direction method. The procedure was initiated via dissection of the inferior pulmonary ligament. Then, the segmental structures were dissected and managed from superficial to deep in order of their appearance: vein, bronchus, artery, and intersegmental planes. The whole procedure proceeded in a single direction. V10. Vein of the dorsal basal segment. B10. Bronchus of the dorsal basal segment. A10. Artery of the dorsal basal segment.

and 2 cases of hamartoma. For patients with primary lung cancer, systemic hilar and mediastinal lymph node sampling was performed in 13 cases, systemic hilar and mediastinal lymph node dissection was performed in 55 cases, and lobe-specific hilar and mediastinal lymph node dissection was performed in 65 cases. Two patients were identified to have lymph node involvement postoperatively ( 1 case each of N1a and N2a1). Both patients refused adjuvant therapy and received regular follow-up. The extent of lymphadenectomy differed among patients because of the difference in their clinical staging and the surgeons' choice. No recurrence or mortality was observed during the median follow-up period of 15 months (range, 1-53 months). The patient with metastatic adenocystic carcinoma did not develop any recurrence after 37 months of follow-up, and the patient with metastatic laryngeal SCC was still disease-free 17 months after the operation. Recurrence-free survival times of 7 and 24 months were demonstrated in the 2 patients with N1a and N2a1, respectively, until the last follow-up in September 2019.

\section{DISCUSSION}

It is widely believed that pulmonary function can be preserved to a greater degree by combining sublobar resection with less invasive thoracoscopic surgery. ${ }^{22}$ Reports with encouraging outcomes from sublobar resection for the treatment of small-sized, noninvasive or minimally invasive lung cancer have been increasing. ${ }^{3,4,6,23-25}$ Although wedge resection is also increasingly considered to be sufficiently effective in
TABLE 1. Patients' characteristics

\begin{tabular}{lc}
\hline \multicolumn{1}{c}{ Characteristic } & Total \\
\hline Age (y) & $\mathbf{N}=\mathbf{1 3 7}$ \\
Range & $26-81$ \\
Median & 50 \\
Male/female & $43 / 94$ \\
Chief symptom & \\
No symptom & 112 \\
Dry cough & 11 \\
Cough with sputum & 8 \\
Chest pain & 4 \\
Chest distress & 2 \\
Comorbidity & \\
Hypertension & 21 \\
Diabetes & 11 \\
Chronic hepatitis B & 4 \\
Arrhythmia & 3 \\
Coronary heart disease & 2 \\
Hyperthyroidism & 1 \\
Solar dermatitis & 1 \\
History of malignancy & \\
Lung cancer & 11 \\
Thyroid cancer & 4 \\
Laryngeal cancer & 2 \\
Breast cancer & 2 \\
Rectal cancer & 1 \\
Adenocystic carcinoma of the maxillary sinus & \\
\hline & \\
\hline & \\
\hline
\end{tabular}

patients with radiologically noninvasive tumors, ${ }^{26-28}$ we considered segmentectomy as the preferred option before the final results of the randomized clinical studies published. ${ }^{7,8}$ During our practice, anatomic segmentectomy is not just a conceptual procedure. When needed, a combined segmentectomy was also performed to ensure sufficient surgical margins according to different features and localization of the tumor. In this study, 47 patients underwent combined segmentectomy.

Thoracoscopic segmentectomies are broadly classified into typical and atypical cases based on the complexity and difficulty of the procedure. ${ }^{29}$ Typical segmentectomies include trisegmentectomy (left S1-3), lingulectomy $(\mathrm{S} 4+5)$, superior segmentectomy (S6), and basilar segmentectomy (S7-10). These cases are not prone to difficulties pertaining to anatomic variations or the identification of intersegmental planes and can be easily performed. In contrast, atypical segmentectomies include segmentectomies of individual segments of the upper lobe, middle lobe, or basal segments. It is especially challenging to perform basal segmentectomies because segmental structures are deeply located in the parenchyma, anatomic variations are common, and the division of additional intersegmental planes is required. As is 
TABLE 2. Surgical types of basal segmentectomy

\begin{tabular}{|c|c|}
\hline Basal segmentectomy & No. of cases \\
\hline Right S7 & 1 \\
\hline Right S8 & 29 \\
\hline Right S9 & 9 \\
\hline Right S10 & 7 \\
\hline Right S7+8 & 3 \\
\hline Right S8+9 & 11 \\
\hline Right S6+7a & 1 \\
\hline Right $\mathrm{S} 6+8$ & 1 \\
\hline Right S6+10 & 2 \\
\hline Right S7+10 & 1 \\
\hline Right S9+10 & 7 \\
\hline Right $S 6+7+8$ & 1 \\
\hline Right S7+8+9 & 2 \\
\hline Right S6+9+10 & 4 \\
\hline Left S8 & 28 \\
\hline Left S9 & 3 \\
\hline Left S10 & 13 \\
\hline Left S6+8 & 1 \\
\hline Left S8+9 & 2 \\
\hline Left S9+10 & 11 \\
\hline
\end{tabular}

commonly found, segmentectomies of right $\mathrm{S} 7$, right $\mathrm{S} 8$, or left S8 may be easily approached through the interlobar fissures when the fissures are well developed. However, these segments also seem to be hard to access when the interlobar fissures are hypoplastic. In addition, segmentectomies of $\mathrm{S} 9, \mathrm{~S} 10$, or $\mathrm{S} 9+10$ are considered the most challenging.

Several methods have been introduced for performing thoracoscopic S10 or S9+10 segmentectomies. One method was to expose the hilum of the basal segments by creating a tunnel between S6 and the basal segments. ${ }^{12}$ However, dividing the lung parenchyma and creating the tunnel between $\mathrm{S} 6$ and the basal segments were technique-demanding procedures, especially when the interlobar fissures were not complete. There is also concern regarding the possible torsion of S6 after complete separation from other segments. In addition, splitting of the parenchyma might result in additional invasiveness and the development of air leakage. Endoh and colleagues ${ }^{11}$ reported a posterior approach for $\mathrm{S} 10$ or $\mathrm{S} 9+10$ segmentectomy. They divided the intersegmental plane between S6 and S10 using the intersegmental veins V6b and V6c as landmarks. Then, the bronchus (B10 or $\mathrm{B} 9+10)$ was exposed followed by dissection of the artery (A10 or A9+10), which was located just behind the bronchus. This method allowed segmentectomy of $\mathrm{S} 10$ or $\mathrm{S} 9+10$ to be performed without the need for dissecting the fissures or separating the S6 segment entirely. A nearly identical
TABLE 3. Perioperative outcomes

\begin{tabular}{|c|c|}
\hline Variables & $\begin{array}{c}\text { Total } \\
\mathbf{N}=\mathbf{1 3 7}\end{array}$ \\
\hline \multicolumn{2}{|l|}{ Operative time $(\min )$} \\
\hline Range & $52-237$ \\
\hline Median & 125 \\
\hline \multicolumn{2}{|l|}{ Blood loss (mL) } \\
\hline Range & $5-250$ \\
\hline Median & 30 \\
\hline \multicolumn{2}{|l|}{ Chest tube duration (d) } \\
\hline Range & $1-22$ \\
\hline Median & 2 \\
\hline \multicolumn{2}{|l|}{ Postoperative hospital stay (d) } \\
\hline Range & $2-24$ \\
\hline Median & 4 \\
\hline \multicolumn{2}{|l|}{ Postoperative complications } \\
\hline Prolonged air leakage $(>5 \mathrm{~d})$ & 5 \\
\hline Prolonged air leakage ( $>5 \mathrm{~d}$ ) and pneumonia & 2 \\
\hline \multicolumn{2}{|l|}{ Tumor size $(\mathrm{cm})$} \\
\hline Range & $0.5-2.7$ \\
\hline Median & 1.0 \\
\hline \multicolumn{2}{|l|}{ Histologic subtypes } \\
\hline $\mathrm{AC}$ & 132 \\
\hline AIS & 14 \\
\hline MIA & 66 \\
\hline Invasive AC & 52 \\
\hline SCC & 1 \\
\hline Metastatic adenocystic carcinoma & 1 \\
\hline Metastatic laryngeal SCC & 1 \\
\hline Hamartoma & 2 \\
\hline \multicolumn{2}{|l|}{ Pathological TNM stage of lung cancer (133 cases) } \\
\hline 0 & 14 \\
\hline IAI1 & 86 \\
\hline IA2 & 21 \\
\hline IA3 & 2 \\
\hline IB & 8 \\
\hline IIB & 1 \\
\hline IIIA & 1 \\
\hline
\end{tabular}

$\overline{A C \text {, Adenocarcinoma; AIS, adenocarcinoma in situ; MIA, minimally invasive }}$ adenocarcinoma; SCC, squamous cell carcinoma.

method called the "bidirectional" approach was introduced by Sato and colleagues ${ }^{14}$ for S10 segmentectomy and its variants. However, these 2 methods do not apply to single S9 segmentectomy or S8 segmentectomy with hypoplastic fissures. The segmental structures in these cases are not directly accessible through an anterior approach starting from the interlobar fissure or a posterior approach. Another method was reported for segmentectomies of S9, S10, or both through a pulmonary ligament approach via tracking the intersegmental septums. ${ }^{10}$ However, the pulmonary ligament is not always contiguous with the intersegmental septum in every case.

Considering the known fissureless techniques used during thoracoscopic lobectomy, we developed a single- 
direction method that permits dissecting the structures in order of their appearance and avoiding division of the fissures. ${ }^{15}$ In our opinion, anatomic segmentectomy can be considered a lobectomy with deeper localized hilar structures and completely hypoplastic fissures. ${ }^{16,17,30}$ In the single-direction manner, it is possible to accomplish all cases of single or combined basal segmentectomy through an inferior pulmonary ligament approach, which is the preferred approach in our practice, without worrying about the fissures. ${ }^{16,17}$ However, when the interlobar fissure is complete, segmentectomy for right $\mathrm{S} 7$, right $\mathrm{S} 8$, or left S8 can be approached easily from the fissure. At that time, either approach can be chosen, depending only on the surgeon's choice. In this study, 33 cases were performed through the interlobar fissure approach.

Before commencing a basal segmentectomy, preoperative planning through image studies is crucial. HRCT or 3-dimensional reconstruction is helpful for the surgeon to decide which segment should be resected and to know the anatomic features of the target segmental structures and its neighbors. When approaching through the inferior pulmonary ligament, a complete picture of the hilum can be achieved from an inferior view. Then, the veins and bronchus of S6, which are normally isolated and located at most posterior and superior, are identified first. After that, both the stems and branches of the common basal segmental veins and bronchi can be confirmed. The main principle for dealing with veins is to divide the branches entering the target segment directly while preserving the intersegmental veins. As the positional relationships of the stem and branches are already known from preoperative planning, the target segmental bronchus can easily be tracked using the "stem-branch" method. ${ }^{17}$ Generally, the branches of S7 (right side) or S8 (left side) are located in the front, the branch of S9 is located in the middle, and the branch of S10 is always the most posterior one. During segmentectomies for $\mathrm{S} 9$ or right $\mathrm{S} 8$, the preserved venous branches are always in the way when we try to dissect the bronchus. A robber belt can be used to retract the venous branches to facilitate exposure of the bronchi. The feeding artery is easily identified because it always runs along the bronchus. The inflation-deflation technique is used to determine the intersegmental planes. For most cases, an obvious inflation-deflation line can be achieved by simply inflating the preserved lung. Occasionally, cross-ventilation across segments does exist and the inflation-deflation line may be vague, especially in patients with emphysema. In such situations, a modified inflationdeflation method can be used. ${ }^{31}$ The whole lung is re-expanded completely with controlled airway pressure under $20 \mathrm{cmH}_{2} \mathrm{O}$. Under such a circumstance, the inflation of the segment to be resected occurs after division of the target bronchus due to cross-ventilation, but deflation is delayed by the fact that the bronchus prevents rapid egress of air. Approximately 10 to 15 minutes later, the preserved segments are deflated, but the target segment is kept expanded so that the demarcation line can be clearly identified. A stapler-based method is used to manage the intersegmental planes, as reported by other surgeons. ${ }^{32}$ In general, the single-direction method allows identification and management of the bronchovascular structures from superficial to deep upon their appearance while avoiding the troublesome dissection of a hypoplastic fissure and inessential splitting of the parenchyma.

In this study, all basal segmentectomies were successfully performed using this single-direction method without conversion to a lobectomy or an open thoracotomy. Thirty-two cases of single S9 (12 cases) or S10 (20 cases) segmentectomies, which were considered the most challenging ones, were successfully completed. There were wide ranges for some of the perioperative morbidities listed. However, 36 patients underwent combined surgeries, which led to longer operative time, drainage duration, and hospital stay. Nevertheless, the overall perioperative outcomes were similar to or even better than those of other reports..$^{10,11,32,33}$ There were 2 patients with unexpected lymph node involvement. We did not perform reoperation because of their compromised physical status. We mainly limited the indication of segmentectomy for GGO-dominant lung cancer and pulmonary metastasis. In addition, we routinely sent station 13 lymph nodes for intraoperative frozen-section examination.

\section{Study Limitations}

This study is a retrospective one without comparisons of techniques. However, because the experience with thoracoscopic basal segmentectomy is still lacking worldwide, we believe that it is still of importance to provide an additional option for thoracic surgeons to consider. We are willing to begin a comparative study in the near future to delineate the merits of this single-direction method. In addition, considering that there are different types of segmentectomies with varied complexities and difficulties, it is hard for us to depict a learning curve. Finally, the follow-up period of patients with lung cancer was short, and long-term outcomes were not observed.

\section{CONCLUSIONS}

The single-direction method for thoracoscopic single or combined basal segmentectomy was feasible and safe in our experience. This method exposes the targeted vessels and bronchi from superficial to deep in order of their appearance without repeated turnover of the lung, and it enables anatomic basal segmentectomy to be performed in a simple manner while avoiding dissection of a hypoplastic fissure or inessential splitting of the pulmonary parenchyma. 


\section{Conflict of Interest Statement}

Authors have nothing to disclose with regard to commercial support.

\section{References}

1. Aberle DR, Adams AM, Berg CD, Black WC, Clapp JD, Fagerstrom RM, et al. Reduced lung-cancer mortality with low-dose computed tomographic screening. N Engl J Med. 2011;365:395-409.

2. Tsutani Y, Miyata Y, Nakayama H, Okumura S, Adachi S, Yoshimura M, et al. Appropriate sublobar resection choice for ground glass opacity-dominant clinical stage IA lung adenocarcinoma: wedge resection or segmentectomy. Chest. 2014; 145:66-71.

3. Tsutani Y, Miyata Y, Nakayama H, Okumura S, Adachi S, Yoshimura M, et al Sublobar resection for lung adenocarcinoma meeting node-negative criteria on preoperative imaging. Ann Thorac Surg. 2014;97:1701-7.

4. Landreneau RJ, Normolle DP, Christie NA, Awais O, Wizorek JJ, Abbas G, et al. Recurrence and survival outcomes after anatomic segmentectomy versus lobectomy for clinical stage I non-small-cell lung cancer: a propensitymatched analysis. J Clin Oncol. 2014;32:2449-55.

5. Hennon M, Landreneau RJ. Role of segmentectomy in treatment of early-stage non-small cell lung cancer. Ann Surg Oncol. 2018;25:59-63.

6. Nomori H, Mori T, Shiraishi A, Fujino K, Sato Y, Ito T, et al. Long-term prognosis after segmentectomy for cT1 N0 M0 non-small cell lung cancer. Ann Thorac Surg. 2019;107:1500-6.

7. Suzuki K, Saji H, Aokage K, Watanabe SI, Okada M, Mizusawa J, et al. Comparison of pulmonary segmentectomy and lobectomy: safety results of a randomized trial. J Thorac Cardiovasc Surg. 2019;158:895-907.

8. Altorki NK, Wang $\mathrm{X}$, Wigle $\mathrm{D}$, Gu L, Darling G, Ashrafi AS, et al. Perioperative mortality and morbidity after sublobar versus lobar resection for early-stage non-small-cell lung cancer: post-hoc analysis of an international, randomised, phase 3 trial (CALGB/Alliance 140503). Lancet Respir Med. 2018:6:915-24.

9. Yan TD. Surgical atlas of thoracoscopic lobectomy and segmentectomy. Ann Cardiothorac Surg. 2014;3:183-91.

10. Kikkawa T, Kanzaki M, Isaka T, Onuki T. Complete thoracoscopic S9 or S10 segmentectomy through a pulmonary ligament approach. J Thorac Cardiovasc Surg. 2015;149:937-9.

11. Endoh M, Oizumi H, Kato H, Suzuki J, Watarai H, Masaoka T, et al. Posterior approach to thoracoscopic pulmonary segmentectomy of the dorsal basal segment: a single-institute retrospective review. J Thorac Cardiovasc Surg. 2017;154:1432-9.

12. Igai H, Kamiyoshihara M, Kawatani N, Ibe T. Thoracoscopic lateral and posterior basal (S9 + 10) segmentectomy using intersegmental tunnelling. Eur J Cardiothorac Surg. 2017;51:790-1.

13. Shimizu K, Nagashima T, Yajima T, Ohtaki Y, Obayashi K, Nakazawa S, et al. Thoracoscopic medial-basal segment segmentectomy. Ann Thorac Surg. 2017; 104:e403-6.

14. Sato M, Murayama T, Nakajima J. Thoracoscopic stapler-based "bidirectional" segmentectomy for posterior basal segment (S10) and its variants. J Thorac Dis. 2018;10:S1179-86.

15. Liu L, Che G, Pu Q, Ma L, Wu Y, Kan Q, et al. A new concept of endoscopic lung cancer resection: single-direction thoracoscopic lobectomy. Surg Oncol. 2010; 19:e71-7.
16. Zhu Y, Pu Q, Liu L. Trans-inferior-pulmonary-ligament VATS basal segmentectomy: application of single-direction strategy in segmentectomy of left S9+10. J Thorac Dis. 2018;10:6266-8.

17. Pu Q, Liu C, Guo C, Mei J, Liu L. Stem-branch: a novel method for tracking the anatomy during thoracoscopic S9-10 segmentectomy. Ann Thorac Surg. 2019; 108:e333-5.

18. Travis WD, Brambilla E, Noguchi M, Nicholson AG, Geisinger KR, Yatabe Y, et al. International Association for the Study of Lung Cancer/American Thoracic Society/European Respiratory Society International Multidisciplinary Classification of Lung Adenocarcinoma. J Thorac Oncol. 2011;6:244-85.

19. Detterbeck FC, Boffa DJ, Kim AW, Tanoue LT. The eighth edition lung cancer stage classification. Chest. 2017;151:193-203.

20. Lin Y, Liu L, Pu Q. Mini-invasive diagnosis and synchronous treatment of solitary pulmonary nodule. Asian Cardiovasc Thorac Ann. 2013;21:306-12.

21. Liu C, Liu L. Reappraise the advanced technique for tumor localization and sentinel lymph node assessment in clinical early-stage non-small cell lung cancer. J Thorac Cardiovasc Surg. 2017;154:1134.

22. Charloux A, Quoix E. Lung segmentectomy: does it offer a real functional benefit over lobectomy? Eur Respir Rev. 2017;26:170079.

23. Zhao ZR, Situ DR, Lau RWH, Mok TSK, Chen GG, Underwood MJ, et al. Comparison of segmentectomy and lobectomy in stage IA adenocarcinomas. J Thorac Oncol. 2017;12:890-6.

24. Lex JR, Naidu B. In patients with resectable non-small-cell lung cancer, is videoassisted thoracoscopic segmentectomy an appropriate alternative to video-assisted thoracoscopic lobectomy? Interact Cardiovasc Thorac Surg. 2016;23:826-31.

25. Kodama K, Higashiyama M, Okami J, Tokunaga T, Imamura F, Nakayama T, et al. Oncologic outcomes of segmentectomy versus lobectomy for clinical T1a N0 M0 non-small cell lung cancer. Ann Thorac Surg. 2016;101:504-11.

26. Altorki NK, Kamel MK, Narula N, Ghaly G, Nasar A, Rahouma M, et al. Anatomical segmentectomy and wedge resections are associated with comparable outcomes for patients with small cT1N0 non-small cell lung cancer. J Thorac Oncol. 2016;11:1984-92.

27. Cho JH, Choi YS, Kim J, Kim HK, Zo JI, Shim YM. Long-term outcomes of wedge resection for pulmonary ground-glass opacity nodules. Ann Thorac Surg. 2015;99:218-22.

28. Yoshida J, Ishii G, Hishida T, Aokage K, Tsuboi M, Ito H, et al. Limited resection trial for pulmonary ground-glass opacity nodules: case selection based on high-resolution computed tomography-interim results. Jpn J Clin Oncol. 2015; 45:677-81.

29. Nakazawa S, Shimizu K, Mogi A, Kuwano H. VATS segmentectomy: past, present, and future. Gen Thorac Cardiovasc Surg. 2018;66:81-90.

30. Zhu Y, Mei J, Liu L. The application of a single-direction strategy in VATS segmentectomy: left S3 segmentectomy. Ann Transl Med. 2018;6:410.

31. Wang J, Xu X, Wen W, Wu W, Zhu Q, Chen L. Modified method for distinguishing the intersegmental border for lung segmentectomy. Thorac Cancer. 2018;9:330-3.

32. Yamanashi K, Okumura N, Otsuki Y, Matsuoka T. Stapler-based thoracoscopic basilar segmentectomy. Ann Thorac Surg. 2017;104:e399-402.

33. Hamada A, Oizumi H, Kato H, Suzuki J, Nakahashi K, Sho R, et al. Learning curve for port-access thoracoscopic anatomic lung segmentectomy. J Thorac Cardiovasc Surg. 2018;156:1995-2003.

Key Words: lung cancer, segmentectomy, single direction, thoracoscopic 